\title{
Verzeichnis der früheisenzeitlichen Fundstellen mit Quellennachweis
}

\begin{tabular}{|c|c|c|c|}
\hline Fundort & Gebiet & Rajon & Literatur/Quelle \\
\hline $\begin{array}{l}\text { IK 5km SW von Žalauly } \\
\text { (I курган 5км C3 } \\
\text { с.Жалаулы) }\end{array}$ & Almaty & Rajymbekskij & \\
\hline $\begin{array}{l}\text { 2K 40om W von Žalauly } \\
\text { (2 кургана } 400 \mathrm{M} 3 \\
\text { с.Жалаулы) }\end{array}$ & Almaty & Rajymbekskij & Bajpakov/Gorjačev 2007, 6I \\
\hline $\begin{array}{l}\text { 3K 7km W von Saty } \\
\text { (3 кургана 7км } 3 \text { с.Саты) }\end{array}$ & Almaty & Rajymbekskij & $\begin{array}{l}\text { Mar'jašev/Gorjačev I99I, 25; } \\
\text { Bajpakov u.a. 2006, I29 }\end{array}$ \\
\hline Gf. Aksaj 9 (Аксай 9) & Almaty & Rajymbekskij & Bajpakov u.a. $2006,26 I-262$ \\
\hline Gf. Akšoky 4 (Акшокы 4) & Almaty & Rajymbekskij & Bajpakov/Gorjačev 2007, 57 \\
\hline Gf. Aktasty 3 (Актасты 3) & Almaty & Rajymbekskij & Bajpakov/Gorjačev 2007, I52-I53 \\
\hline Gf. Asy Saga (Асы Зага) & Almaty & Enbekšikazachskij & $\begin{array}{l}\text { Grošev/Mar'jašev I992, I3; } \\
\text { Nagler 2009, 408, Abb. 76; } \\
\text { Samašev u.a. 2009, 350-352; } \\
\text { Nagler u.a. 2010, 49-54; } \\
\text { Gass 20IIa, 65-67, Fig. I4-22; } \\
\text { Gass 20I1b, 2I2-215, Abb. 5-8, I0-I4; } \\
\text { Gass, im Druck }\end{array}$ \\
\hline $\begin{array}{l}\text { Gf. am Stausee Bartogaj (Бар- } \\
\text { тогайское водохранилище) }\end{array}$ & Almaty & Enbekšikazachskij & \\
\hline Gf. Bes Tobe (Бес Тобе) & Almaty & Enbekšikazachskij & Mar'jašev u.a. 1986,29 \\
\hline Gf. Bestobe 3 (Бестобе 3) & Almaty & Rajymbekskij & Bajpakov/Gorjačev 2007, 77 \\
\hline Gf. Boroldaj (Боролдай) & Almaty & Stadt Almaty & $\begin{array}{l}\text { Tujakbaeva u.a. I985, I0; } \\
\text { Samašev u.a. 2005e, 73; } \\
\text { Samašev u.a. 2006; } \\
\text { Gass 20IIb, } 215\end{array}$ \\
\hline Gf. Ereul (Ереул) & Almaty & Rajymbekskij & Bajpakov/Gorjačev 2007, I77-I78, I80-I92 \\
\hline Gf. Issyk (Иссык) & Almaty & Enbekšikazachskij & $\begin{array}{l}\text { Bernštam I938, I9; } \\
\text { Dublickij I939a, I/93/8I; } \\
\text { Bernštam I94Ib, 6, 27; } \\
\text { Archeologičeskaja karta Kazachstana I960, 309-310; } \\
\text { Čarikov I960; } \\
\text { Maksimova I97I; } \\
\text { Akišev K.A. I97I; } \\
\text { Artamonov I973, 36, 38; } \\
\text { Akišev K.A. I974, 6I-77; } \\
\text { Akišev K.A. I978; } \\
\text { Akišev A.K. I980a; } \\
\text { Livšic I980, 3-I2; } \\
\text { Bajpakov I992, 56; } \\
\text { Nurmuchanbetov u.a. I992, 29, 35; } \\
\text { Zadneprovskij I992, 77-78; } \\
\text { Bajpakov u.a. I993, I09; } \\
\text { Bajpakov/Bektureeva I998, 2-I4; } \\
\text { Zagorodnij/Grigor'ev I998, II7-I24; } \\
\text { Parzinger 2004, 56-6I; } \\
\text { Samašev u.a. 2004b; } \\
\text { Bajpakov u.a. 2005c, 2-3, 6-8, 22-3I; }\end{array}$ \\
\hline
\end{tabular}




\begin{tabular}{|c|c|c|c|}
\hline Fundort & Gebiet & Rajon & Literatur/ Quelle \\
\hline & & & $\begin{array}{l}\text { Samašev u.a. 2005a, 38, 40, 49, 86-89, II2; } \\
\text { Parzinger 2006, 659-662; } \\
\text { Samašev 2007, I65-I67; } \\
\text { Baipakov 2008, 58; } \\
\text { Martynov A. 2008, I0; } \\
\text { Nagler 2008, 70, 73; } \\
\text { Ongar 2008, 22I, 223; } \\
\text { Gass 20IIa, 60-6I, Fig. 5-7; } \\
\text { Gass 20IIb, 2II-2I2, Abb. 2-4; } \\
\text { Gass, im Druck }\end{array}$ \\
\hline $\begin{array}{l}\text { Kurganketten auf dem Plateau } \\
\text { Kegen } \\
\text { (Плато Кеген) }\end{array}$ & Almaty & Rajymbekskij & $\begin{array}{l}\text { Turkestan I9I4, I23-I24; } \\
\text { Bartol'd I966, 77-78; } \\
\text { Akišev K.A. I974, 6I; } \\
\text { Otčety otdelov i ekspedicij I979, 33; } \\
\text { Nagler 2009, 408, Abb. 77; } \\
\text { Nagler u.a. 20I0, 49-54; } \\
\text { Gass 20IIb, 2I5-2I6, Abb. I5-2I; } \\
\text { Gass, im Druck }\end{array}$ \\
\hline Gf. Kokpijaz (Кокпияз) & Almaty & Rajymbekskij & \\
\hline $\begin{array}{l}\text { Kurgangruppe Krasnyj Vostok } \\
\text { (группа курганов у } \\
\text { с.Красный Восток) }\end{array}$ & Almaty & Enbekšikazachskij & \\
\hline $\begin{array}{l}\text { Gf. Kyzylšaryk (Кызыл } \\
\text { шарык) }\end{array}$ & Almaty & Enbekšikazachskij & Grošev/Mar'jašev I992, I2 \\
\hline $\begin{array}{l}\text { Gf. Nordwestlicher Friedhof } \\
\text { (Северо-западное клад- } \\
\text { бище) }\end{array}$ & Almaty & Stadt Almaty & Samašev u.a. 2005 a, I0o \\
\hline $\begin{array}{l}\text { Gf. Novoalekseevka (Новоа- } \\
\text { лексеевка) }\end{array}$ & Almaty & Enbekšikazachskij & $\begin{array}{l}\text { Kopylov I956, 4-2I; } \\
\text { Ageeva I957, II; } \\
\text { Kopylov I958, I58-I77; } \\
\text { Archeologičeskaja karta Kazachstana I960, 308; } \\
\text { Akišev K.A. I974, 6I; } \\
\text { Bajpakov u.a. 2005b, 5-I9; } \\
\text { Samašev u.a. 2005a, 38; } \\
\text { Ongar 2008, 221, } 224\end{array}$ \\
\hline $\begin{array}{l}\text { Kurgankette Sarytau „A“ (Cap- } \\
\text { ытау «A») }\end{array}$ & Almaty & Rajymbekskij & \\
\hline Gf. Saty (Саты) & Almaty & Rajymbekskij & \\
\hline Gf. Šyrganak 2 (Шырганак 2) & Almaty & Rajymbekskij & Bajpakov u.a. 2006, 224-227 \\
\hline Gf. Taučilik-6 (Таучилик-6) & Almaty & Rajymbekskij & Bajpakov u.a. 2006, I53 \\
\hline Gf. Togyzbulak (Тогызбулак) & Almaty & Rajymbekskij & \\
\hline
\end{tabular}




\begin{tabular}{|c|c|c|c|}
\hline Fundort & Gebiet & Rajon & Literatur/Quelle \\
\hline Gf. Turgen (Турген) & Almaty & Enbekšikazachskij & $\begin{array}{l}\text { Bernštam/Dublickij I936, 5-6; } \\
\text { Bernštam I938, I9; } \\
\text { Dublickij I939a, I-8, I/93/8I; } \\
\text { Bernštam I94Ib, 6, 27; } \\
\text { Dublickij I946a, I-I6; } \\
\text { Bernštam I948a, 8I; } \\
\text { Archeologičeskaja karta Kazachstana I960, 308; } \\
\text { Akišev K.A. I974, 6I; } \\
\text { Bajpakov I992, 56; } \\
\text { Nurmuchanbetov u.a. I992, 3, 36, Abb. 5; } \\
\text { Nurmuchanbetov I998, 27-50, Abb. I-7; } \\
\text { Parzinger 2004, 56; } \\
\text { Ongar 2008, 22I; } \\
\text { Mar'jašev/Gorjačev 2009a, 9-10, I5-24, 29-32; } \\
\text { Mar'jašev/Gorjačev 2009b, 7-8, Foto I3-I4; } \\
\text { Gass 20IIa, 6I-65, Fig. 8-13 }\end{array}$ \\
\hline Gf. Ulžan (Улжан) & Almaty & Stadt Almaty & $\begin{array}{l}\text { Samašev u.a. 2005a, I0I; } \\
\text { Nurpeisov u.a. 2008, 225-240 }\end{array}$ \\
\hline Gf. Žalauly 2 (Жалаулы 2) & Almaty & Rajymbekskij & Bajpakov/Gorjačev 2007, 92, 96-98, Abb. 7I, 74-76 \\
\hline Gf. Žalauly 8 (Жалаулы 8) & Almaty & Rajymbekskij & $\begin{array}{l}\text { Bajpakov 2003, IIо, Abb. I; } \\
\text { Bajpakov/Gorjačev } 2007,8 \text { I-82, Abb. 62-63 }\end{array}$ \\
\hline $\begin{array}{l}\text { Gf. Žoan Tobe } \\
\text { (Жоан Тобе) }\end{array}$ & Almaty & Enbekšikazachskij & $\begin{array}{l}\text { Čarikov I956a, 29-36; } \\
\text { Čarikov I956b, 27-55; } \\
\text { Maksimova I956a, 3-8; } \\
\text { Maksimova I956b, 33-42; } \\
\text { Opis' predmetov I956, 8, I3-I4, I8-23, 25-26; } \\
\text { Pacevič I956, 32-38; } \\
\text { Semirečenskaja archeologičeskaja ekspedicija I956, I-I3; } \\
\text { Ageeva I957, 52/6r; } \\
\text { Maksimova I960a, 60-64; } \\
\text { Akišev K.A. I967, 67; } \\
\text { Artamonov I973, 36; } \\
\text { Zadneprovskij I992, 76; } \\
\text { Nagler 2009, 406-408, Abb. 72-75; } \\
\text { Samašev u.a. 2009, 350-352; } \\
\text { Nagler u.a. 20I0, 49-54; } \\
\text { Gass 20IIa, 67-68, Fig. 23; } \\
\text { Gass 20IIb, 213, Abb. 9; } \\
\text { Gass, im Druck }\end{array}$ \\
\hline Gf. Žylysaj I (Жылысай I) & Almaty & Rajymbekskij & Bajpakov u.a. $2006,349,353$ \\
\hline $\begin{array}{l}\text { Komplex Aščibulak } \\
\text { (Ащибулак) }\end{array}$ & Almaty & Enbekšikazachskij & \\
\hline $\begin{array}{l}\text { Komplex Butakty I } \\
\text { (Бутакты I) }\end{array}$ & Almaty & Stadt Almaty & $\begin{array}{l}\text { Mar’jašev/Gorjačev I998, 20, 23, Foto 34; } \\
\text { Mar'jašev/Gorjačev I999a, 20, 23, Foto 92; } \\
\text { Gorjačev 2004, 3, 7-8, I2, 52-58, Foto 7-I6, Abb. 5-24; } \\
\text { Gorjačev 2005, 3, 5, 9-I2, I3I-I45, Abb. 3-5, I4-35, Foto } \\
\text { 35-66; } \\
\text { Baipakov 2008, 8I-88, Abb. 30-32; } \\
\text { Bajpakov u.a. 2008, 3, 5-7, II-23, I75-I78, Foto 22-27, } \\
\text { 62-III, Abb. 3, I8-68; } \\
\text { Bajpakov u.a. 2009, 3, 5-7, I4-20, 23-33; Abb. 5, 26-9I; } \\
\text { Foto I73-I84 }\end{array}$ \\
\hline
\end{tabular}




\begin{tabular}{|c|c|c|c|}
\hline Fundort & Gebiet & Rajon & Literatur/ Quelle \\
\hline Komplex Kiikpaj (Киикпай) & Almaty & Enbekšikazachskij & $\begin{array}{l}\text { Mar'jašev u.a. I986, 29-30; } \\
\text { Nurmuchanbetov/Savel'eva I988, I0-II; } \\
\text { Savel'eva I994, 49-50, Abb. I5; } \\
\text { Bajpakov u.a. 2002, 30, Abb. II-I2; } \\
\text { Gass 20IIb, 2I8, 220, Abb. 28-29; } \\
\text { Gass, im Druck }\end{array}$ \\
\hline Komplex Sarytau (Сарытау) & Almaty & Rajymbekskij & \\
\hline Komplex Turgen 2 (Тургень 2) & Almaty & Enbekšikazachskij & $\begin{array}{l}\text { Mar'jašev/Gorjačev I999a, 3, I4, I6, Foto 63-77, 84; } \\
\text { Abb. 84-87; } \\
\text { Mar'jašev/Gorjačev I999b, 3-4, I6, 28-33, 35-37, 43-44, } \\
\text { 46-55, Abb. 6o-73, 82-87, Foto 65-83; } \\
\text { Gorjačev 200Ib, 4, 6, I9-24, Abb. 26-32, 39-43; Foto } \\
\text { 37-48; } \\
\text { Mar'jašev/Gorjačev 200I, II7, I2I; } \\
\text { Gorjačev 2002, 3, 5-8, 23, 37-38, Abb. 3, Foto I-4; } \\
\text { Gorjačev 2003, 3, 5, 9-II, Abb. II-I2, I4-I8; } \\
\text { Gorjačev 2004, 3, 20, 33-35, 45, 47-51, Abb. I08-II8; Foto } \\
\text { I89-I90, I93-I96; } \\
\text { Gorjačev 2007b; } \\
\text { Mar'jašev/Gorjačev 2008, 3, 7-8, 29-34, I52-I55, I58-16o, } \\
\text { Abb. 58-72, Foto 79-II0; } \\
\text { Mar'jašev/Gorjačev 2009a, 3-4, 6-7, 49-60, 64-67, } \\
\text { Abb. 39-56, Foto 49-99; } \\
\text { Mar'jašev/Gorjačev 2009b, 3-4, I0-36, Abb. 7-67, Foto } \\
\text { 28-IoI }\end{array}$ \\
\hline Sdl. Aktasty (Актасты) & Almaty & Rajymbekskij & \\
\hline Sdl. Bachtijar (Бахтияр) & Almaty & Enbekšikazachskij & \\
\hline Sdl. Cyganka 8 (Цыганка 8) & Almaty & Talgarskij & $\begin{array}{l}\text { Bajpakov/Chang 2000, 266-268; } \\
\text { Bajpakov u.a. 2000b, I9; } \\
\text { Chang u.a. 2003, 30I-309; } \\
\text { Samašev u.a. 2005a, 52-53, I28-I29; } \\
\text { Baipakov 2008, 77-79, Abb. 28-29 }\end{array}$ \\
\hline $\begin{array}{l}\text { Sdl. bei der Stadt Issyk (y } \\
\text { г.Иссык) }\end{array}$ & Almaty & Enbekšikazachskij & $\begin{array}{l}\text { Gass 20IIb, 2I8; } \\
\text { Gass, im Druck }\end{array}$ \\
\hline $\begin{array}{l}\text { Sdl./Issyk „Tagebaustätte beim } \\
\text { Geburtshaus“ (Карьер у } \\
\text { роддома, г.Иссык) }\end{array}$ & Almaty & Enbekšikazachskij & Gass 20IIb, 2I 8 \\
\hline $\begin{array}{l}\text { Sdl./Issyk „Zentraler Friedhof“ } \\
\text { (Центральное кладбище, } \\
\text { г.Иссык) }\end{array}$ & Almaty & Enbekšikazachskij & Gass 20IIb, 2I 8 \\
\hline Sdl. Junki г (Юнки I) & Almaty & Enbekšikazachskij & \\
\hline Sdl. Kajnazar (Кайназар) & Almaty & Enbekšikazachskij & $\begin{array}{l}\text { Nurmuchanbetov/Savel'eva I988, II-I3; } \\
\text { Savel'eva I994, 34-35, 89-90, 93-II5, I26, Abb. II, 35,4 und } \\
40, \mathrm{I} \text {; } \\
\text { Bajpakov u.a. 2002, 28-29 }\end{array}$ \\
\hline $\begin{array}{l}\text { Sdl./Turtkul Karakemer } \\
\text { (Каракемер) }\end{array}$ & Almaty & Enbekšikazachskij & $\begin{array}{l}\text { Dublickij I939a, 2; } \\
\text { Bernštam I94ıb, 6; } \\
\text { Ageeva I96ob, 2; } \\
\text { Savel'eva I994, 48; } \\
\text { Bajpakov u.a. 2002, 30; } \\
\text { Gass 20Iıb, Abb. 22 }\end{array}$ \\
\hline $\begin{array}{l}\text { Sdl. Kotlovandy } \\
\text { (Котлованды) }\end{array}$ & Almaty & Enbekšikazachskij & $\begin{array}{l}\text { Bajpakov u.a. 2005a, 223-224; } \\
\text { Mar'jašev/Gorjačev 2009b, Foto } 7\end{array}$ \\
\hline
\end{tabular}




\begin{tabular}{|c|c|c|c|}
\hline Fundort & Gebiet & Rajon & Literatur/ Quelle \\
\hline $\begin{array}{l}\text { Sdl. Krasnyj Vostok (Красный } \\
\text { Восток) }\end{array}$ & Almaty & Enbekšikazachskij & $\begin{array}{l}\text { Savel'eva I994, 47-48; } \\
\text { Bajpakov/Nurmuchanbetov 2000, I4; } \\
\text { Gass 20IIb, 219, Abb. 25-27; } \\
\text { Gass, im Druck }\end{array}$ \\
\hline $\begin{array}{l}\text { Sdl. Kyzylbulak } 2 \\
\text { (Кызылбулак 2) }\end{array}$ & Almaty & Enbekšikazachskij & $\begin{array}{l}\text { Gorjačev 2004, 20; } \\
\text { Bajpakov u.a. 2005a, 202-203; } \\
\text { Mar'jašev/Gorjačev 2008, } 6\end{array}$ \\
\hline $\begin{array}{l}\text { Sdl. Kyzyl-Gajrat } \\
\text { (Кызыл-Гайрат) }\end{array}$ & Almaty & Talgarskij & $\begin{array}{l}\text { Savel'eva I994, 35; } \\
\text { Bajpakov u.a. 2002, } 29\end{array}$ \\
\hline Sdl. Rachat (Рахат) & Almaty & Enbekšikazachskij & $\begin{array}{l}\text { Bajpakov/Nurmuchanbetov 2000, I4-I6, Abb. 2; } \\
\text { Bajpakov u.a. 2005c, 4-5, I2-2I; } \\
\text { Baipakov 2008, 7I-72, Abb. I6-I8; } \\
\text { Gass 20IIb, 2I8-2I9, Abb. 23-24; } \\
\text { Gass, im Druck }\end{array}$ \\
\hline Sdl. Sarytau (Сарытау) & Almaty & Rajymbekskij & Gass 20IIb, 22I-222, Abb. 30-3I \\
\hline Sdl. Talgar (Талгар) & Almaty & Talgarskij & $\begin{array}{l}\text { Gorodeckij I924, 68/II8/, Nr. 20; } \\
\text { Bernštam I938, I9; } \\
\text { Bernštam I94Ib, 3-5, 24-25, 30; } \\
\text { Bernštam I946, IIo; } \\
\text { Dublickij I946b, I4; } \\
\text { Kopylov I956, I9-33, Taf. 8-I5; } \\
\text { Ageeva I957, 9-II, 55/64-57/66; } \\
\text { Ageeva I96ob, 2, 9; } \\
\text { Kopylov I962; } \\
\text { Kopylov I963; } \\
\text { Kopylov I965; } \\
\text { Kopylov I966; } \\
\text { Kopylov I978, 6I-65; } \\
\text { Otčety otdelov i ekspedicij I979, 30-32; } \\
\text { Otčety o rabote I98I, 35; } \\
\text { Kopylov I982; } \\
\text { Kopylov I983; } \\
\text { Kopylov I984; } \\
\text { Kopylov I985; } \\
\text { Kopylov I986; } \\
\text { Kopylov I987; } \\
\text { Savel'eva I989, 432-438; } \\
\text { Kopylov/Kerekeša I993, I22-I36; } \\
\text { Savel'eva I993; } \\
\text { Savel'eva I994, 2I-33, 62-8I, 88-II5, I25, I28-I30, } \\
\text { Abb. 2-I0, 35,I und 35,2, 39; } \\
\text { Savel'eva/Vojakin I999, 68-75; } \\
\text { Bajpakov u.a. 200ob, I9; } \\
\text { Chang/Tourtellotte 2000, 83-85; } \\
\text { Korženkov u.a. 20oI, I0I-I02; } \\
\text { Bajpakov u.a. 2002, 8-28, 60-I49, I88-209; } \\
\text { Savel'eva/Vojakin 2002, I46-I55; } \\
\text { Bajpakov u.a. 2005d, 2-3 }\end{array}$ \\
\hline
\end{tabular}




\begin{tabular}{|l|l|l|l|}
\hline Fundort & Gebiet & Rajon & Literatur/ Quelle \\
\hline Sdl. Tuzusaj (Тузусай) & Almaty & Talgarskij & Tujakbaeva u.a. I993; \\
& & Tujakbaeva u.a. I994; \\
& & Bajpakov I995, 40-65, Abb. I-II; \\
& & Kuznecova I995, 58-65; \\
& & Bajpakov I997, 3, 47-56, 58-67; \\
& & Chang/Grigoriev I999, 39I-4I0; \\
& & Grigor'ev/Ismagil I999, 87; \\
& & Bajpakov/Nurmuchanbetov 2000, 6-II; \\
& & Bajpakov u.a. 2000b, I9; \\
& & Chang/Tourtellotte 2000, 83-85; \\
& & Rosen u.a. 2000, 6II-623; \\
& & Bajpakov u.a. 2002, 9; \\
& & Chang u.a. 2003, 30I-309; \\
& & Samašev u.a. 2005a, 52-53, I26-I27; \\
& & Parzinger 2006, 793; \\
& & Grigor'ev u.a. 2007a, I29-I39; \\
& & Baipakov 2008, 74-77, Abb. 22-23 \\
& &
\end{tabular}

